\title{
Global Climate Change and the Resurgence of Tropical Disease: An Economic Approach*
}

\author{
Douglas Gollin \\ Williams College
}

\author{
Christian Zimmermann \\ University of Connecticut
}

June 17, 2010

\begin{abstract}
We study the impact of global climate change on the prevalence of tropical diseases using a heterogeous agent dynamic general equilibrium model. In our framework, households can take actions (e.g., purchasing bednets or other goods) that provide partial protection from disease. However, these actions are costly and households face borrowing constraints. Parameterizing the model, we explore the impact of a worldwide temperature increase of $3^{\circ} \mathrm{C}$. We find that the impact on disease prevalence and especially output should be modest and can be mitigated by improvements in protection efficacy.
\end{abstract}

*Authors' addresses: Douglas Gollin, Department of Economics, Williams College, 24 Hopkins Hall Drive, Williamstown, MA 01267 (dgollin@williams.edu); Christian Zimmermann, Department of Economics, Department of Economics, University of Connecticut, 341 Mansfield Road, Unit 1063, Storrs, CT 06269 (christian.zimmermann@uconn.edu). This paper was written while Gollin was on leave at the Yale School of Forestry and Environmental Studies. 


\section{Introduction}

Public health officials often use the term "tropical diseases" to refer collectively to a list of infectious diseases that are found primarily in developing countries. These include malaria, schistosomiasis, dengue, trypanosomiasis, leprosy, cholera, and leishmaniasis, among others. Many of these diseases are spread by insect vectors, and all of them disproportionately affect the world's poor. Malaria is the most severe of these, with the World Health Organization (WHO 2008a) estimating that the disease causes about 250 million episodes of "acute illness" and perhaps 880,000 deaths annually. ${ }^{1}$ But other diseases, less well known and sometimes described as "neglected," also impose grave burdens on people living in the tropics. The World Health Organization estimates that "neglected tropical diseases" affect over one billion people each year and cause about 570,000 deaths annually (World Health Organization 2009). For example, some 200 million people are currently infected with schistosomiasis, a parasite that is transmitted through poor sanitation. ${ }^{2}$ Perhaps 50 million cases of dengue occur each year, of which 500,000 lead to devastating hemorrhagic fever, with 22,000 resulting deaths. $^{3}$

What makes these diseases "tropical"? The diseases themselves are perfectly capable of infecting people in all climates, but they are transmitted by vectors that are most common in hot environments: mosquitoes (malaria and dengue); biting flies (trypanosomiasis and Chagas disease); sandflies (leishmaniasis); and freshwater snails (schistosomiasis). As Masters and McMillan (2001) have pointed out, most of these vectors do not fare well in temperate zones that are susceptible to frost. Regular seasonal frost does not eliminate the vectors, but it does appear to interfere with cycles of transmission, making it harder for the diseases to sustain high levels of infection.

If cold weather is responsible for suppressing the vectors that transmit tropical diseases, how will climate change alter the current distribution of these diseases? Will we see malaria and other tropical diseases sweeping through rich countries? At

\footnotetext{
${ }^{1}$ Reported by WHO on the "Roll Back Malaria" program website at: http://malaria.who.int/wmr2008/malaria2008.pdf.

${ }^{2}$ Of these, several million display serious health consequences. (Source: http://www.who.int/schistosomiasis/en/index.html.)

${ }^{3}$ Dengue is also perhaps the fastest-growing of the tropical diseases, with a recent dramatic increase in the number of cases observed around the world. See http://www.who.int/csr/disease/dengue/impact/en/ for further information.
} 
present, infectious disease is a relatively minor source of the disease burden in today's rich temperate-zone countries. Will this change in future?

The Intergovernmental Panel on Climate Change (IPCC) has specifically investigated the changing burdens of infectious disease that may result from climate change. One IPCC background report notes that:

Climate plays a dominant role in determining the distribution and abundance of insects and tick species - directly, through its effects on vector and parasite development, and indirectly through its effects on host plants and animals and land-use changes.... Therefore, it is anticipated that climate change will have an effect on the geographical range and seasonal activity of vector species and, potentially, disease transmission... ${ }^{4}$

The IPCC studies soberly suggest that the capacity of medical and public health systems in most rich countries are sufficient to prevent tropical diseases from reaching epidemic levels, under the assumed range of warming that is expected to accompany climate change. Not all observers share this confidence, however; one leading scholar argues that "...[A] warming and unstable climate is playing an ever increasing role in driving the global emergence, resurgence, and redistribution of infectious diseases" (Epstein 2004, p. 383).

This paper specifically takes up the question of how climate change might be expected to alter the distribution and severity of tropical diseases? Will increasing temperatures lead to the spread of mosquitoes and other insect vectors and thereby expand infectious diseases to the previously "safe" countries of the temperate zone? Or will the biological impacts of climate change be mitigated by human adaptation and behavioral responses? If both effects are present, how will they be balanced?

We view these questions as fundamentally related to human behavioral choices at the individual level as well as at the level of government policies. Although climate change may affect the spatial distribution of vectors and pathogens, human exposure to disease is not a mechanical function of environmental conditions. In many or most tropical diseases, either the vector or the pathogen must spend at least a portion of its lifecycle in humans. This means that humans affect the level of disease prevalence through their choices about prevention and treatment of the diseases.

\footnotetext{
${ }^{4}$ See: http://www.ipcc.ch/ipccreports/tar/wg2/index.php?idp $=507$.
} 
Given that human choices are critical, we approach our questions from the perspective of economists. We use a model in which individuals make conscious and rational choices that affect their disease exposure and infection status. Individual choices may not be socially optimal: there are infection externalities operating in this environment. Nevertheless, we begin by considering the competitive equilibrium of a dynamic general equilibrium model in which people face a risk of infection from diseases in each period. People in the model economy make choices that affect their exposure to the disease, as in the real world they may buy drugs, bednets, or other goods that allow them to prevent or treat the diseases that they face.

To assess the overall impact of climate change on disease prevalence, we consider the effects of changes in the parameter that describes the "ecology of disease" in the model. In effect, we make disease transmission easier. We then ask how this alters levels of infection and also the levels of steady-state output in the model economy.

Our paper is organized as follows. Section 2 presents a simple model of malaria that incorporates important features of both economic and epidemiological models. Section 3 parameterizes, or "calibrates" this model to match some central features of the data. Section 4 describes the results of several experiments undertaken using the model economy. Finally, Section 5 concludes.

\section{The Model}

A useful model of climate change and its impact on disease must have certain features. For example, it must represent infection through some plausible process. Disease should affect people's choices and perhaps also their resources and abilities. And it is useful to consider actions that people can take that alter their exposure to the disease - either by avoiding infection or by treating (or curing) infections once they occur. In short, to understand the impact of climate change on the prevalence of a human disease, we think it is essential to take into account how people react and do something about the environment they live in. Thus, a model needs the following components:

1. agents that react to the disease and economic environment;

2. an economic environment that is influenced by the prevalence of the disease; 
3. a law of motion for the disease that is at least partially influenced by humans;

4. economic constraints that potentially prevent an eradication of the disease;

5. heterogeneity among agents, as only some carry the disease, and not all face the same economic constraints;

6. general equilibrium effects, as the prevalence of the disease does not only affect the ailing agents, but also the healthy ones through prices and potential exposure to the disease.

Thus, we use a dynamic stochastic general equilibrium model with heterogeneous agents, following Gollin and Zimmermann (2010). This is a model of perpetual youth, where agents stochastically catch a disease, and die with probabilities that depend on their health status. The likelihood of falling sick depends on the proportion of people already sick, as well as on ecological factors that can change with climate change. Once sick, people remain so for the rest of their lives and suffer productivity losses because of absences from work or diminished abilities.

However, economic agents have access to a technology that can at least partially protect them from getting infected. This technology is costly and needs to be paid for in lump sum fashion before it is used. This might correspond to a vaccine, or alternatively a long-lasting bednet or a set of screens for a house. The fixed cost of this preventive measure poses a problem for individuals in the economy, as agents do not necessarily have the funds available for this purchase, especially at the beginning of their economic life. Indeed, we assume that borrowing is not possible in this economy.

Agents earn income in two ways. First, they work and obtain a wage that corresponds to their marginal productivity of labor. The latter is influenced by their health status, an idiosyncratic and persistent productivity shock and the overall marginal productivity of labor. Second, they earn interest on their savings, at the overall marginal productivity of capital. Factor prices are determined from a production function that uses aggregates of efficient labor units and savings across the entire model economy.

We now turn to the detailed description of this economy, starting with workers. There is a unit measure of them and they maximize their lifetime expected discounted 
utility subject to a budget constraint and a liquidity contraint:

$$
\begin{aligned}
\max _{\left\{c_{i t}, k_{i, t+1}\right\}_{t=0}^{\infty}} & \sum_{t=0}^{\infty} \beta^{t}\left(1-d\left(h_{i t}\right)\right) u\left(c_{i t}\right), \\
\text { s.t. } & c_{i t}+k_{i, t+1}+p_{i t} q \leq w_{t} h_{i t} \pi_{i t}+r_{t} k_{i t}, \quad \forall t, \\
& k_{i t}>0, \quad \forall t,
\end{aligned}
$$

where, for agent $i$ in period $t, c_{i t}$ is consumption, $k_{i t}$ is capital, $p_{i t}$ is a binary decision to purchase protection against the disease, $h_{i t}$ is the health status and $\pi_{i t}$ is an idiosyncratic productivity shock. $u(\cdot)$ is a utility function with the usual properties, $\beta$ is the discount rate, $d(\cdot)$ is the probability of death, $q$ is the price of health protection, $w_{t}$ is the efficiency wage and $r_{t}$ is the return of capital. Idiosyncratic productivity shocks $\pi_{i t}$ follow a persistent binomial Markov process.

Individuals are born healthy and without assets, replacing those who just died. Within each period, an unprotected individual may catch a disease with probability $s_{t}$. Being sick is an absorbing state and entails a loss in productivity, so that $h_{i t}=\bar{h}<1$ instead of $h_{i t}=1$.

We now turn to aggregates. Efficiency units of labor and individual capital are aggregated and used in a standard production function:

$$
\begin{aligned}
L_{t} & =\sum_{i} h_{i t} \pi_{i t}, \\
K_{t} & =\sum_{i} k_{i t}, \\
Y_{t} & =F\left(K_{t}, L_{t}\right) .
\end{aligned}
$$

Similarly, the prevalence of the disease is measured by the proportion of sick people:

$$
S_{t}=\sum_{i} \mathbb{I}_{h_{i t}=\bar{h}}
$$

This translates into the probability of catching the disease $s_{t}$ by combining $S_{t}$ with an ecological factor $Z$ and the efficacy of protection $e$, if purchased $\left(v_{i t}=1\right)$ :

$$
s_{i t}= \begin{cases}\left(\left(1-v_{i t}\right)+v_{i t} e\right) f\left(Z, S_{t}\right) & \text { if } f\left(Z, S_{t}\right)>\bar{s} \\ 0 & \text { if } f\left(Z, S_{t}\right) \leq \bar{s}\end{cases}
$$


Note that below a threshold $\bar{s}$, a disease is not sustainable and vanishes.

We solve this model economy by restricting ourselves to steady states. The recursive steady state equilibrium is defined by decisions for consumption, capital and protection purchase, prices for labor and capital, distributions of agents over health status and capital holdings, aggregate labor, capital and disease prevalence such that individuals maximize their problem given prices and aggregates, firms likewise maximize profits, factors markets are efficients, distributions are ergodic and individual decisions are consistent with aggregates.

There is always at least one equilibrium, a disease-free one. If the threshold $\bar{s}=0$, there is another one with some disease prevalence. Indeed, even if everyone buys protection, newborns can still catch a disease in the first period and thus perpetuate it. If the threshold is higher, such an equilibrium may not exist.

\section{Calibration}

As we are interested in quantitative answers, we need to calibrate this model economy; i.e., we must select functional forms and find values for all parameters. In most respects, we follow Gollin and Zimmermann (2010), which offers a more detailed explanation of the calibration.

First, we set the functional forms. For the production function, we use the standard Cobb-Douglas formulation:

$$
Y_{t}=K_{t}^{\alpha} L_{t}^{1-\alpha}
$$

Similarly, for the utility function, why use a standard power function:

$$
u\left(c_{i t}\right)=\frac{\left(\gamma c_{i t}\right)^{1-\rho}}{1-\rho}
$$

where $\gamma$ is a multiplier that captures the value of life. Indeed, without it, utility may be negative and thus agents may prefer death, which has zero utility.

Finally, we need a functional form to transform disease prevalence into a proba- 
bility of catching the disease, assuming no protection is obtained:

$$
f\left(Z, S_{t}\right)=Z S_{t}^{\mu}
$$

with both $Z$ and $S_{t}$ between zero and one and $\mu$ strictly positive.

We now turn to putting numbers to parameters. We assume a periodicity of a year. Thus we set $\beta$ at 0.95 and $\rho=1$, which corresponds to logarithmic utility. To set $\gamma$, we observe from Viscusi and Aldy (2003) that the value of life in the US is about $\$ 7$ million, or 11.3 times lifetime consumption. Thus we set $\gamma=11.3$.

For the productivity loss due to sickness, we set it at $10 \%$ following Bleakley's work on malaria (2003). This is a middle-of-the-road estimate from a wide distribution of micro studies in the literature. For some other diseases, infected people may be severely debilitated and may suffer from greater productivity losses. Others, because they cause only sporadic ill effects, may have smaller effects on lifetime income. Thus $\bar{h}=0.9 .^{5}$

We use Domeij and Heathcote (2004) to establish the binary process for the idiosyncratic productivity shocks $\pi_{i t}$. Shocks are $\pm 22.4 \%$, with a $90 \%$ chance of repeating the following year. The annual death rate is set to $1.5 \%$ for healthy people and $7.5 \%$ for sick people. The fertility rate is set to keep population constant, as required by a steady-state. This is also consistent with observed positive correlations between fertility and mortality rates.

Regarding the protection against the disease, we need to establish its efficacy and its cost. Note again that we model the preventive good as something that is purchased one time and then provides continued protection for a lifetime. Given the borrowing constraint, this would appear to impose strong limitations to the availability of protection. Yet Gollin and Zimmermann (2010) show that this cost must rise to 1.5 times the average annual income to prevent everyone in the economy from purchasing prevention as soon as they can possibly afford it. Thus, people in this economy display a strong desire to protect themselves from disease. This desire would be even greater if we increased the productivity losses associated with sickness.

\footnotetext{
${ }^{5}$ Note that setting the productivity loss higher does not necessarily increase the impact of the disease in equilibrium; instead, as the disease becomes more costly to individuals, there are greater incentives for people to purchase protection. In equilibrium, this may decrease the fraction of the population infected, leading to a reduction in the aggregate costs of the disease.
} 
Taking into account the strong demand for prevention, we set the cost of the preventive good at one quarter of the annual average income in a disease free economy, with the understanding that considerable variation in this cost will not affect results. Although people do not need to purchase the preventive good again, the protection that it provides may be imperfect. Vaccines are not always effective, and mosquito nets or screens provide only partial protection from disease. In our model, we note that a mosquito net reduces the probability of catching malaria by $70 \%$. Combining this with other methods may increase it further, but would still keep it far from $100 \%$. For example, a proposed malaria vaccine is expected to have an efficacy of only $50 \%$. Thus, we explore different values for the efficacy of protection between $70 \%$ and $90 \%$.

Next, we need to calibrate the parameters for the infection rate process. We use the malaria-ecology index of Sachs et al. (2004), rescaled to the unit interval, to

measure $Z$. The elasticity $\mu$ is found by regressing observed infection rates on the malaria ecology. We find $\mu=0.122$.

Lastly, we have to determine the prevalence threshold that enables a natural eradication of the disease, $\bar{s}$. Unfortunately, the literature is not clear about whether eradication is feasible. In the case of malaria, for example, few are willing to commit to any hard numbers. Those that do disagree: WHO (2008b) claims a country may be ready for a concerted eradication effort if the annual infection rate falls below $0.1 \%$. Others claim it needs to be significantly lower than that. Because of this uncertainty, we consider a range of different values, starting with a value of zero (no threshold effect).

\section{Quantitative Analysis and Results}

In our framework, an increase in temperature will be equivalent to an increase in the malaria ecology parameter, $Z$. In the original index developed by Sachs et al. (2004), temperature is one of several variables used to construct the index value. When we compare our re-scaled measure of $Z$ for different countries to the average annual temperature average annual temperature from 1972-2001, we find a strong positive relationship between temperature and the malaria ecology for countries with values 
Figure 1: Temperature and Malaria Ecology, Cross-Section Data

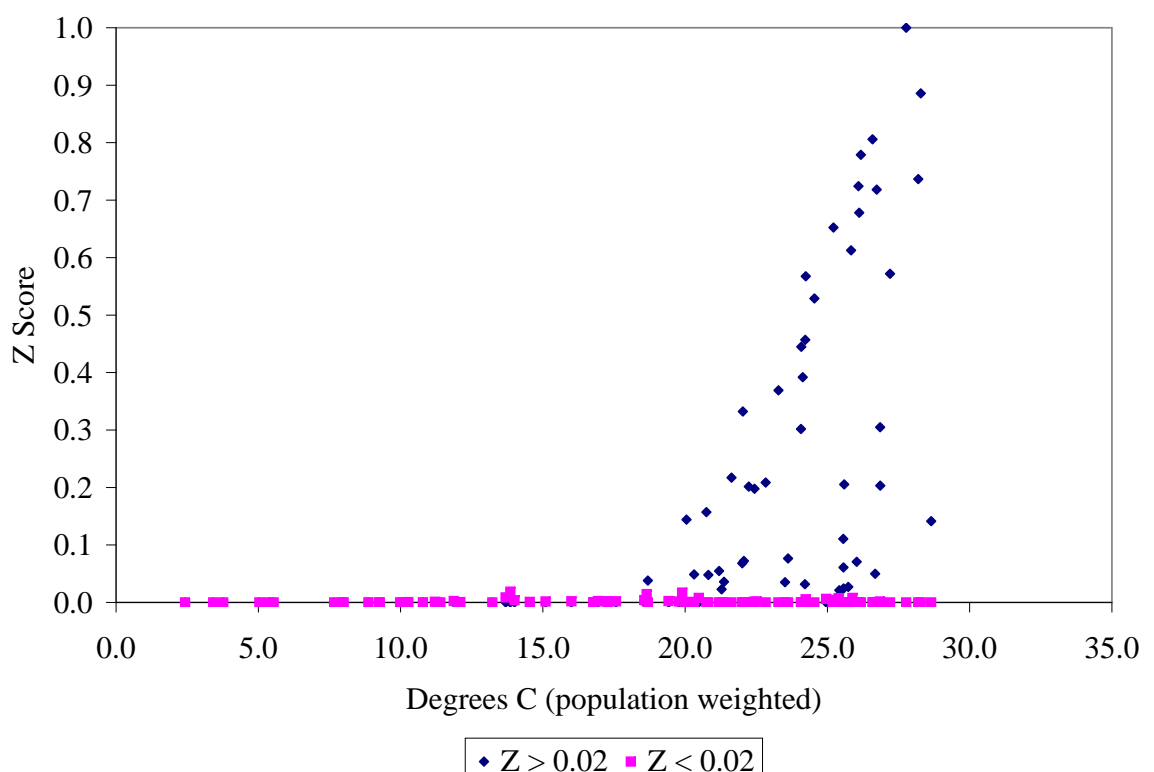

of $Z>0.3$. (See Figure 1.) ${ }^{6}$

Thus, we simulate the effect of climate change by solving the model economy as calibrated above for different values of $Z$ between 0.3 and 1.0 and for different degrees of protection efficacy. In this context, efficacy refers to the ability of the protective bundle to provide protection from the disease. Perfect efficacy of $100 \%$ would imply that someone who purchased the protective bundle would never get infected; efficacy of $90 \%$ implies that someone purchasing the protective bundle faces a $90 \%$ reduction

\footnotetext{
${ }^{6}$ Data on temperature were taken from the appendix to Jones and Olken (2010), which are available online at: Climate Shocks and Exports with Ben Jones. American Economic Review Papers and Proceedings, forthcoming 2010. Data and programs at: http://econ-www.mit.edu/files/5131. The temperatures used here are based on detailed observations for different geographic cells within countries; the country averages are based on population-weighted averages of these temperatures.
} 
in the probability of infection; and so on. In these scenarios, we consider protection efficacies of $70 \%, 80 \%$ and $90 \% .^{7}$ The results are summarized in Figure (2) for output and Figure (3) for the proportion of sick people.

For output, we see in Figure 2 that for a given level of protection efficacy, a higher level of $Z$ implies lower steady-state output per person; hence, each of the curves shown in the figure is downward sloping. We also see that higher levels of efficacy lead to improvements in output per person (shifting the curves as illustrated). We represent climate change here as a movement along an iso-efficacy curve. The figure suggests that the consequences of climate change may be relatively modest in this model economy. Similar patterns are evident when we look at the effects of an increase in temperature on disease prevalence. Figure 3 shows the relationship between infection rates and $Z$ for the same three different levels of efficacy.

What do these numbers mean for climate change of the magnitudes currently predicted by climate models? Climate change is estimated to increase average world temperature by 1 to $6^{\circ} \mathrm{C}$. Taking $3^{\circ} \mathrm{C}$ as a benchmark, this would effectively give New York City a climate like that of present-day Washington, DC; Rome would see its average annual temperature rise to that of present-day Algiers. In terms of malaria ecology, such a temperature change would correspond to an increase in $Z$ of 0.192 , based on the relationship shown in Figure $1 .^{8}$

Table 1 shows that the impact of climate change through disease is relatively modest. We consider three scenarios that differ in terms of the efficacy of the protective good. The change in output corresponds to less than a year in real GDP growth. The change in the number of sick people is minimal (it is a percentage of a percentage). Locally the impact could be stronger or reversed if one factors in changes in rainfall, which are predicted to increase or decrease depending on location.

Why is the impact so small? For one, temperature is only one factor in malaria ecology. Also, diseases like malaria may not have that much of an aggregate impact

\footnotetext{
${ }^{7}$ At a protection efficacy of $100 \%$, aggregates are unaffected as everybody but a tiny proportion of the population is immune, and changes in the ecological environment have a minimal impact. In any case, $100 \%$ is by far not realistic with current technologies.

${ }^{8}$ Specifically, we used a linear regression of $Z$ on a constant and the average annual temperature variable, measured in degrees $\mathrm{C}$. This yielded a highly significant coefficient on temperature of 0.064 . For a shift of $3^{\circ} \mathrm{C}$, this gives an increase in $Z$ of approximately 0.192 .
} 
Table 1: Impact of a $3^{\circ} \mathrm{C}$ increase in temperature on output and number of sick people (in \%)

\begin{tabular}{|l||cc|}
\hline Protection efficacy & output & sick \\
\hline \hline $90 \%$ & $-0.40--0.96$ & $+0.65-+2.22$ \\
$80 \%$ & $-0.26--1.22$ & $+0.43-+1.99$ \\
$70 \%$ & $-0.13--1.03$ & $+0.32-+1.62$ \\
\hline
\end{tabular}

on rich economies, as our previous research has suggested, because people are likely to pay for protection. Only if the protective technologies are ineffective or if people are simply unable to afford them would we expect to find large economic impacts. ${ }^{9}$

Would climate change make malaria sustainable where it was not before? Seeing the disease prevalence in Figure (3), it seems unlikely that any of the thresholds for eradication would cross any of the curves. While some areas with high malaria potential currently are free from it, for example Singapore, it is not because they are below the threshold. In other words, we do not find that rich temperate-zone countries will avoid the burdens of tropical disease by actually eradicating them; instead, our model suggests that the disease burdens will be very modest because people are able to protect and treat themselves easily. As a result, very few people will ever be infected, and transmission will be very low - though not impossible.

Climate change will increase the burden of tropical diseases in our model. However, rather modest improvements in protection efficacy could compensate for the consequences of climate change. For example, an improvement in efficacy from $70 \%$ to $80 \%$ improves GDP by $6-8 \%$ and decreases the number of sick people by about $10 \%$, much larger effects than those mentioned in Table 1.

\footnotetext{
${ }^{9}$ We note, however, that there may be large welfare impacts even if GDP per capita is not greatly affected.
} 
Figure 2:

Output, no disease threshold, by efficiency

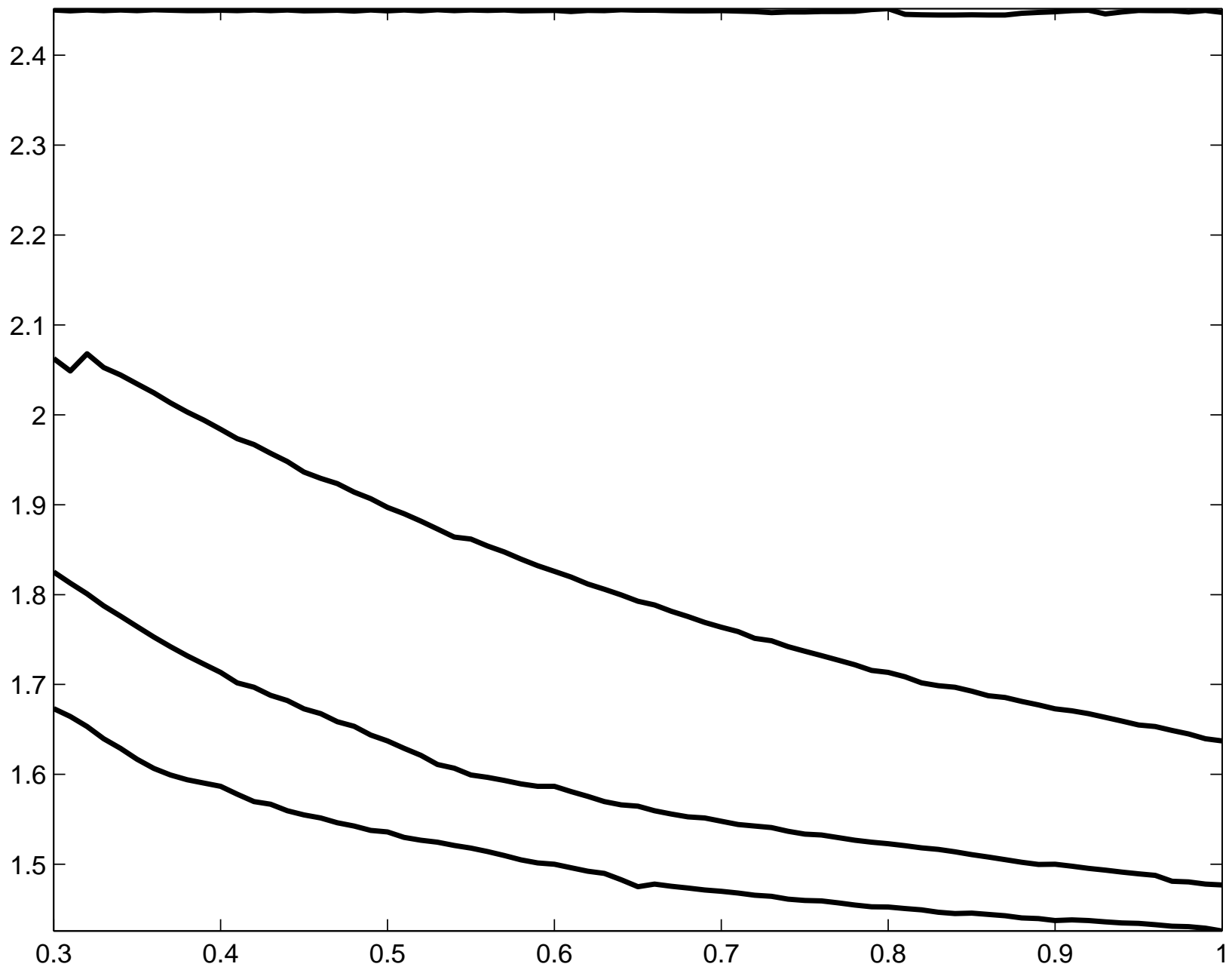

\section{Conclusions}

Will global climate change increase significantly the prevalence of tropical diseases such as malaria? We answered this question using a heterogeneous agent dynamic general equilibrium model where households have the opportunity to react to the prevalence of diseases. We find that a middle-of-the-road scenario with an increase of temperature of $3^{\circ} \mathrm{C}$ would lead to rather modest increase in disease prevalence and 
Figure 3:

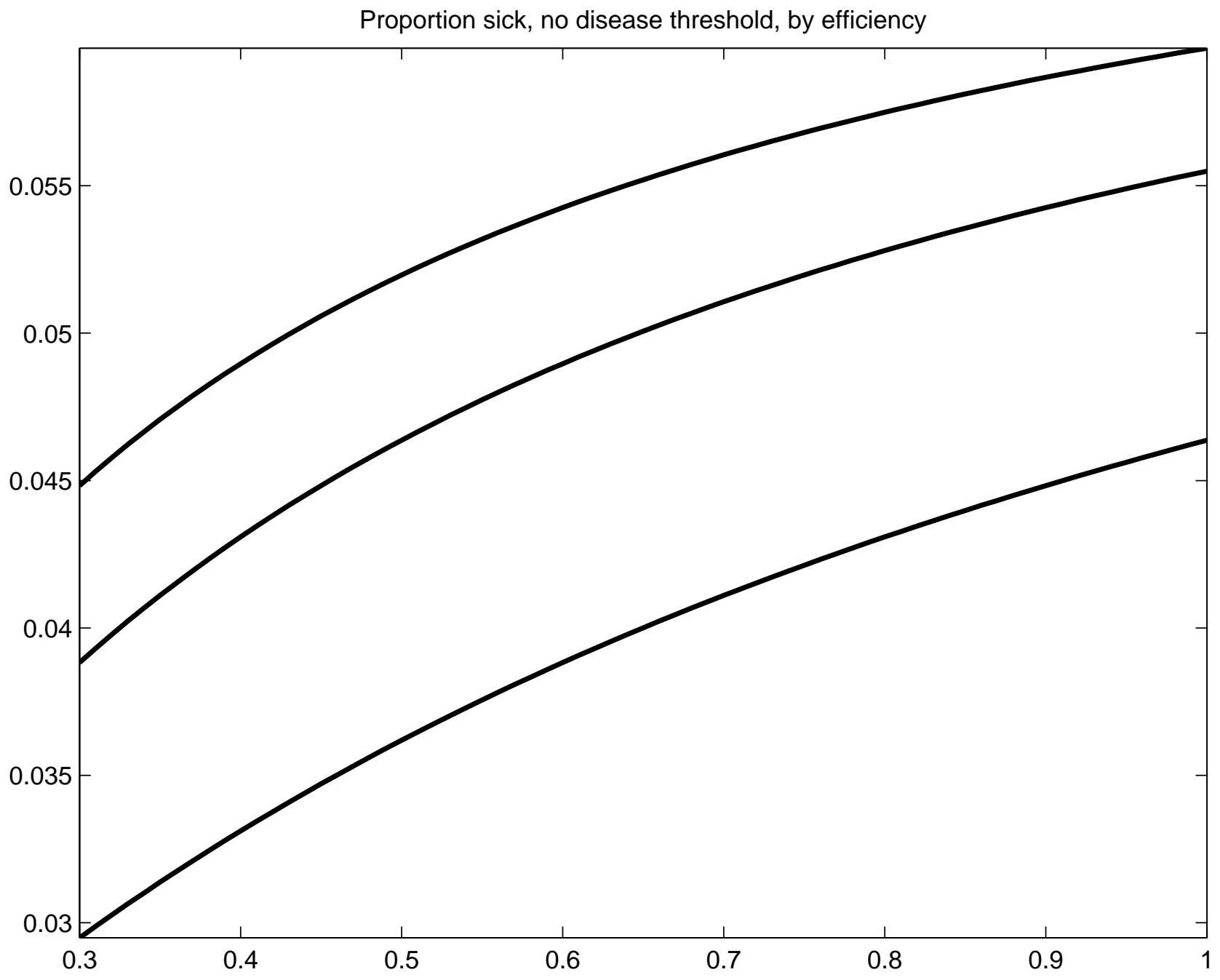


very modest disease-induced losses in GDP per capita. We find that households will elect to acquire protection as soon as they can, typically within the very first years, thus mitigating considerably the impact of global climate change in this respect.

In particular, we find that the cost of protection is generally unimportant: households value life sufficiently to absorb considerable expenses in the name of prophylaxis. A more important factor in the efficacy of protection. With current technology, efficacy between $70 \%$ and $90 \%$ is attainable, and future developments will certainly drive up these figures. The effects of improvements in disease protection will, in this sense, more than compensate for the impact of global climate change. Improvements in the efficacy of disease prophylaxis can potentially have large impacts on welfare. 


\section{References}

Bleakley, Hoyt. 2003. "Disease and Development: Evidence from the American South." Journal of the European Economic Association 1.2-3: 376-86.

Bleakley, Hoyt. 2009. Malaria eradication in the Americas: A retrospective analysis of childhood exposure. Forthcoming, American Economic Journal: Applied.

Domeij, David and Jonathan Heathcote. 2004. On the distributional effects of reducing capital taxes. International Economic Review 45(2): 523-54.

Epstein, Paul R. 2004. Climate Change and Public Health: Emerging Infectious Diseases. Encyclopedia of Energy, Volume 1., pp. 381-92 (Elsevier).

Gollin, Douglas and Christian Zimmermann. 2010. Malaria: Disease impacts and long-run income differences. University of Connecticut, Department of Economics Working Paper 2007-30R, revised.

Jones, Ben and Benjamin Olken. 2010. Climate shocks and exports. American Economic Review Papers and Proceedings, forthcoming.

Masters, William A and Margaret S. McMillan. 2001. Climate and scale in economic growth. Journal of Economic Growth 6(3): 167-86.

Sachs, Jeffrey, Anthony Kiszewski, Andrew Mellinger, Andrew Spielman, Pia Malaney, and Sonia Ehrlich Sachs. 2004. A global index of the stability of malaria transmission. American Journal of Tropical Medicine and Hygiene 70(5): 486-498.

Viscusi, W. Kip and Joseph E. Aldy. 2003. The value of a statistical life: a critical review of market estimates throughout the world. Journal of Risk and Uncertainty 27(1): 5-76.

World Health Organization. 2008a. World Malaria Report 2008. Geneva: WHO.

World Health Organization. 2008b. Global Malaria Control and Elimination: Report of a Technical Review, 17-18 January 2008. Geneva: WHO.

World Health Organization. 2009. Neglected Tropical Diseases, Hidden Successes, Emerging Opportunities. Geneva: WHO. 